\title{
Pupil Center Location Based on Skin Color Segmentation and Radial Symmetry Transform
}

\author{
Wei Wei ${ }^{1, a}$, Yujing Zhou ${ }^{2, b}$, Jingran Zhou ${ }^{3, c}$, Zhenxi Zhang ${ }^{4, d}$ \\ ${ }^{1}$ School of Computer, Electronics and Information, Guangxi University, No.100 Daxue Road \\ Nanning, Guangxi 530004 P.R.CHINA \\ a weiweigxu@qq.com, b992004469@qq.com, ${ }^{\mathrm{c}}$ 1017455201@qq.com, ${ }^{\mathrm{d}}$ 583510863@qq.com
}

\author{
Keywords: Face Detection, Skin Color Segmentation, Background Similar Skin Color Area \\ Removal, Eye Detection. Radial Symmetry Transform
}

\begin{abstract}
To realize the eye pupil center location in color face image, a new algorithm based on skin color segmentation and radial symmetry transform is proposed. The algorithm makes full use of the pupil and the surrounding region's gradient variation characteristics and radial symmetry characteristic, which can effectively solve the interference factors such as uneven spot, frame. Step: First, use the skin color model to detect the face region, and then remove the small block of the background with similar skin color by morphological opening operation and area comparison filtering method. Then, use the horizontal integral projection to determine the candidate eye regions. Finally, apply the radial symmetry transform to the candidate eye region to locate pupil center. Experimental results show that the proposed algorithm has good localization effect, and has strong robustness to various disturbances.
\end{abstract}

\section{Introduction}

In recent years, line of sight tracking technology is an important and active research area of computer vision. It is widely used in human-computer interaction, auxiliary driving vehicles, virtual reality and military fields [1]. An important process in the line of sight tracking system is the extraction and detection of line of sight characteristic parameters. Eyes as the most important visual organ contains a wealth of sight feature parameters such as the pupil center. Therefore, a fast, accurate and effective eye location algorithm is very important.

\section{Proposed Method}

Fig. 1 shows the pupil center location algorithm flow. First, detect face region by the skin color model in the YCbCr color space. Then, using gray level features of eye region, the candidate eye region is detected by the horizontal integral gray projection algorithm [2, 3, 4]. Finally, apply the radial symmetry transform to the candidate eye region to locate pupil center.

Face Detection Based on Skin Color Segmentation. Skin color is one of the most striking features of color face images, which is relatively concentrative and stable, and not affected by the brightness of the image. Skin color has a strong clustering feature. Use the ellipse skin color model proposed by $\mathrm{Hsu}[5]$ based on $\mathrm{YCbCr}$ color space, Where the $\mathrm{Y}$ represents the brightness information, and $\mathrm{Cb}$ and $\mathrm{Cr}$ represent the blue chrominance component the red chrominance component respectively. Ellipse skin color model is shown in Eq.1 and Eq.2, Where $\mathrm{C}_{\mathrm{b}}$ and $\mathrm{C}_{\mathrm{r}}$ represent the original blue component and red component in the YCbCr color space respectively, and $x$ and $y$ represent chrominance components after transformation. Bring X, Y into the left of 
formula (1), if the result is less than or equal to 1 , the pixel belongs to the skin color area. Otherwise, the pixel is not the skin color region. We use elliptical skin color model to make color face image binaryzation. The formula of binarization is Eq.3, Where gray $(x, y)$ repents the gray value of the pixels (x,y), D represents skin color area. The original face image and skin color segmentation result are shown in Fig.2 (a) and Fig. 2(b).

$$
\begin{aligned}
& \frac{\left(x-e c_{x}\right)}{a^{2}}+\frac{\left(y-e c_{y}\right)}{b^{2}}=1 \\
& {\left[\begin{array}{l}
x \\
y
\end{array}\right]=\left[\begin{array}{cc}
\cos \theta & \sin \theta \\
-\sin \theta & \cos \theta
\end{array}\right]\left[\begin{array}{l}
C_{b}^{\prime}-C_{x} \\
C_{r}^{\prime}-C_{y}
\end{array}\right]} \\
& \operatorname{gray}(x, y)=\left\{\begin{array}{cc}
255 & (x, y) \in D \\
0 & (x, y) \notin D
\end{array}\right.
\end{aligned}
$$

Remove the Similar Skin Color Regions in the Background. As shown in Fig2.(b) the similar skin color regions in the background are also detected, which can interfere with the localization of the face region. We use morphological open operation and regional area comparison filtering to remove the background of the similar skin color region. The definition of morphological open operation is Eq.4.

$$
\begin{aligned}
& A \circ B=(A \Theta B) \oplus B \\
& P y(i)=\sum_{x=1}^{N} f(x, i) \quad i=1,2, \ldots, M
\end{aligned}
$$

The formula (4) indicates A made by B morphological open operation. Morphological open operation can smooth the contour of the image, disconnect the narrow connection, and remove the small protruding part [6]. Using the characteristics of morphological open operation, we can eliminate some of the skin color regions in the background. Fig.3 shows the result after morphology opening operation. After the morphological open operation, the image background is still containing some similar skin color region. We use the region marking method to find out the area of the largest area, which is the face region, and then through the area filtering method, filter out the block of similar skin color region in the background smaller than the face region. And then remove the ear area by area filtering method. The Fig. 4 shows the result after the area filtering method, which shows that the image basically only reserves the face region. The upper and lower left and right boundary of the face region are found according to the filtered face image. Finally, the face region in the original image is marked by red rectangle box. Marked result is shown in Fig.5. Fig. 6 shows the face region detected from the original face image.

Eye Candidate Region Detection Based on Gray Level Integration Projection. Apply the horizontal gray integral projection to face region's gray image to detect the eye candidate regions roughly. Set the size of the cropped face image as $\mathrm{M}_{\text {rows }} \times \mathrm{N}_{\text {columns, }}$, then the horizontal integral projection formula which is a sum of pixel value in every line is shown in Eq.5. 


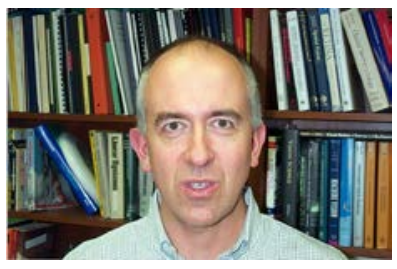

Fig.2(a) Original face image

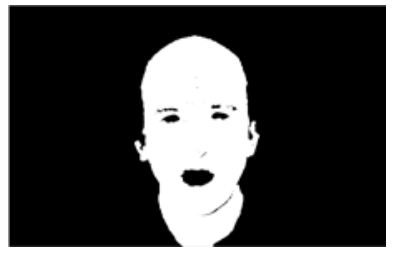

Fig.4. Face image after area comparison filtering

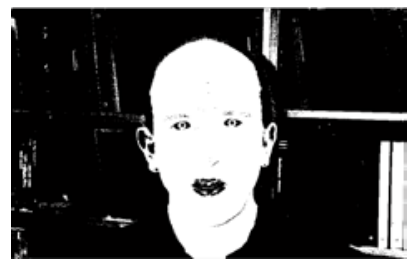

Fig.2(b) Face image after skin color segmentation

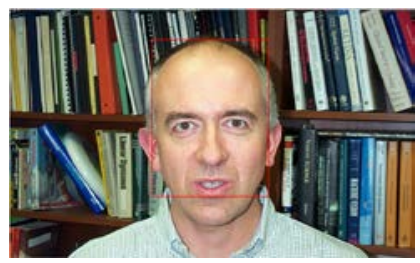

Fig.5. Face region marked by a rectangular box

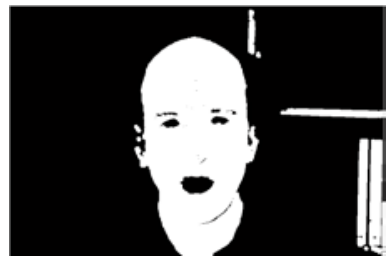

Fig.3.Face image after morphological open operation

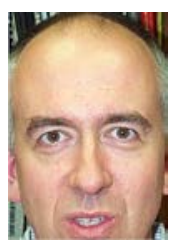

Fig.6.Face region cropped from original face image

The horizontal integral projection curve of the gray face image is shown in Fig.7. Notice that the gray value of the eyebrow region is lower than that of the forehead and the upper eyelid, and the gray value of the pupil and iris are lower than that of the upper eyelid and lower eyelid. According to the gray scale characteristics of the eyebrow and eye regions we can conclude from Fig.7 that the first valley in the projection curve should correspond to the eyebrow area, the second valley value should be eye ball region, and that the larger projection value between first valley value and the second valley value should correspond to the area between the eyebrows and upper eyelashes. Crop the eye region which starts from the area between the eyebrows and the upper eyelashes and ends to the position lower than eye ball 20 pixels. The Cropped eye area is shown in Fig.8.

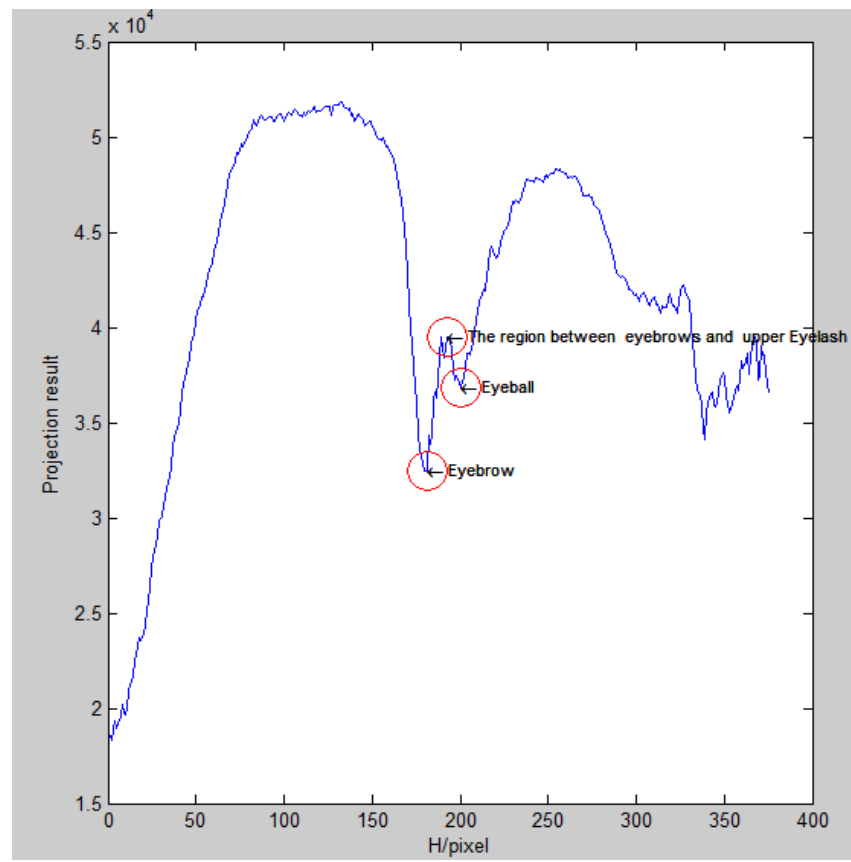

Fig.7.Gray horizontal integration projection of face region The eye region image is divided into two parts with same width, the left eye region and the right eye region. Fig. 9(a) and Fig.9 (b) show results of left and right eyes.

Pupil Center Location Based on Radial Symmetry Transform (RST). Considering that the gray value of the pupil and the iris are much smaller than the other regions, and that the pupil and iris are concentric circle or concentric ellipse, the center of the pupil can be located by the radial symmetry transform (RST). RST proposed by Loy [7] for detecting the region of interest is based on the gradient to consider the radial symmetry contribution of each pixel to its surrounding pixels. Bright spots and dark spots can be detected by using RST in different gradient directions.Fig.10 shows the flow of RST. 


\section{(.) $P(0)$ ?}

Fig.8. Eye region obtained from gray projection of face image

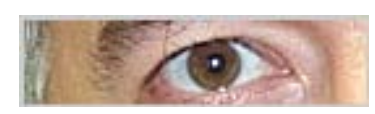

Fig.9(a)Left eye region

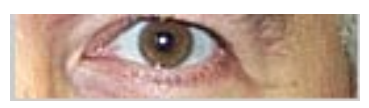

Fig.9(b)Right eye region

In Fig.10 $g$ is the gradient of input image. For each pixel $\boldsymbol{p}$,the gradient $g$ is defined as $\left[g_{\mathrm{x}}(\mathrm{p}), \mathrm{g}_{\mathrm{y}}(\mathrm{p})\right]$. Each pixel point in the direction of the gradient has the positive affected pixel $\boldsymbol{p}_{+\mathrm{ve}}(\boldsymbol{p})$ and the negative affected pixel $\boldsymbol{p}_{\text {-ve }}(\boldsymbol{p})$. The location relationship between $\boldsymbol{p}, \boldsymbol{p}_{\text {+ve }}(\boldsymbol{p})$ and $\boldsymbol{p}_{\text {-ve }}(\boldsymbol{p})$ is shown in Fig.11. $\boldsymbol{P}_{+\mathrm{ve}}(\boldsymbol{p})$ and $\boldsymbol{p}_{\text {-ve }}(\boldsymbol{p})^{\prime}$ 's coordinates are shown in Eq.6 where $n$ is the detection radius. In Fig.10 Mn and On are respectively the gradient direction projection and the gradient magnitude projection. They are defined as Eq.7
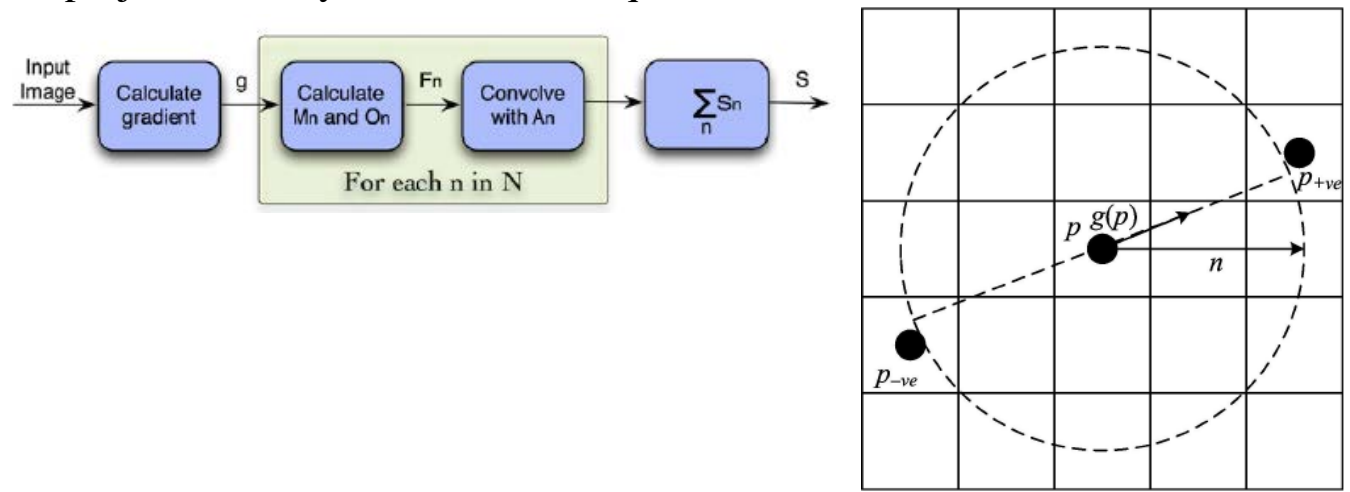

Fig.10. The flowchart of radial symmetry transform algorithm

Fig.11.The location relationship between pixel $\mathrm{p}$, $\mathrm{p}_{+\mathrm{ve}}$ and $\mathrm{p}_{\text {-ve }}$

$$
\left.\mathbf{p}_{ \pm v e}(\mathbf{p})=\mathbf{p} \pm \operatorname{round}\left(\frac{g(\mathbf{p})}{\|g(\mathbf{p})\|} n\right) \quad \text { ('+'represents } \mathbf{p}_{+v e},- \text { 'represents } \mathbf{p}_{-v e}\right)
$$

$$
\begin{aligned}
O_{n}\left(\mathbf{p}_{+v e}(\mathbf{p})\right) & =O_{n}\left(\mathbf{p}_{+v e}(\mathbf{p})\right)+1 \\
O_{n}\left(\mathbf{p}_{-v e}(\mathbf{p})\right) & =O_{n}\left(\mathbf{p}_{-v e}(\mathbf{p})\right)-1 \\
M_{n}\left(\mathbf{p}_{+v e}(\mathbf{p})\right) & =M_{n}\left(\mathbf{p}_{+v e}(\mathbf{p})\right)+\|g(\mathbf{p})\| \\
M_{n}\left(\mathbf{p}_{-v e}(\mathbf{p})\right) & =M_{n}\left(\mathbf{p}_{-v e}(\mathbf{p})\right)-\|g(\mathbf{p})\|
\end{aligned}
$$

When the detection radius is $n$,the result of RST is:

$$
\begin{aligned}
& S_{n}=F_{n} * A_{n} \\
& F_{n}(\mathbf{p})=\frac{M_{n}(\mathbf{p})}{k_{n}}\left(\frac{\left|\tilde{O}_{n}(\mathbf{p})\right|}{k_{n}}\right)^{\alpha}
\end{aligned}
$$

Where $\alpha$ is the radial control parameters, $\mathrm{k}_{\mathrm{n}}$ is a scale factor used to normalize the $\mathrm{M}_{\mathrm{n}}$ and $\mathrm{O}_{\mathrm{n}}$ obtained under different radius, and $\mathrm{A}_{\mathrm{n}}$ is a two-dimensional Gaussian template used to carry out Gaussian smoothing. Finally, the final result of RST is the average of the RST results $\mathrm{S}_{\mathrm{n}}$ obtained through all the detection radius.

$$
S=\frac{1}{|N|} \sum_{n \in N} S_{n}
$$

In this paper, use the RST to locate the pupil center. Gray gradient direction is generally from a low gray toward the high gray. As the region near the edge of the pupil and near the iris edge's 
positive gradient direction is deviated from the center of pupil, the region's negative gradient direction is pointing towards the center of pupil. To detect the pupil center, the algorithm is improved in the process of using the RST and the $\mathrm{M}_{\mathrm{n}}$ and $\mathrm{O}_{\mathrm{n}}$ are only used along the pixel points' negative gradient direction. The final RST result is calculated according to the two projection diagrams. In the process of the RST, with the change of the radius of RST, the edge points of the pupil and the edge of the iris will be superimposed on the nearby the center of the pupil, which will have a maximum value. The position of the maximum value of the RST results is the location of the pupil center. Due to the fact that eyelashes and frame do not have radial symmetry, no matter how the detection radius changes, their results will not produce superposition. Therefore, they will not affect the pupil center detection. Similarly, due to the fact that light spot's high gray value, the negative gradient direction of the light spot's edge points is deviated from the center of a circle, and the RST results along the negative gradient direction will not be superimposed in the center of the light spot. Therefore, light spot will also not affect the pupil center detection.

The RST algorithm is used to locate pupil center having four steps. Step 1, Compute the gradient image of the left/right eye gray level image; Step 2, Set the detection radius $\mathrm{N}_{\min }$ and $\mathrm{N}_{\max }$; Step 3,For $\mathrm{n}=\mathrm{N}_{\min }: 1: \mathrm{N}_{\max }$ : (1)Firstly, calculate the coordinates of the negative affected points along the negative direction by the Eq. 6. (2)Then, calculate $O_{n}\left(\mathbf{p}_{\text {-ve }}(\mathbf{p})\right)$ and $M_{n}\left(\mathbf{p}_{\text {-ve }}(\mathbf{p})\right)$.

(3) Convert the Eq.9 into $F_{n}(\mathbf{p})=\frac{\left|M_{n}(\mathbf{p})\right|}{k_{n 1}}\left(\frac{\left|\tilde{O}_{n}(\mathbf{p})\right|}{k_{n 2}}\right)^{\alpha}$, and calculate Fn(p) where $\alpha=2, k n 1=\max \left(\operatorname{abs}\left(\mathrm{M} \_\mathrm{n}(:)\right)\right), \mathrm{kn} 2=\max \left(\operatorname{abs}\left(\mathrm{O} \_\mathrm{n}(:)\right)\right)$.Calculate the RST result by Eq.8 under detection radius $n$, where Gaussian window's variance and size are respectively $0.1 n$ and $\operatorname{ceil}(n / 2) \times \operatorname{ceil}(n / 2)$. Step 4, Calculate $S$ by Eq.10, and find out the coordinates of the maximum $S_{\max }$, which corresponds to the pupil center's location.

The RST results of the left and right eyes are respectively shown in Fig.12.

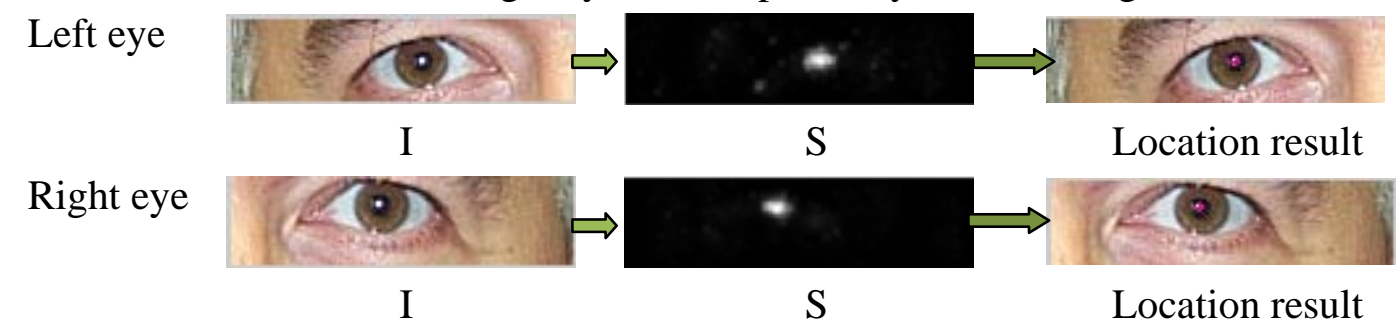

Fig.12.RST results

\section{Experimental Results and Discussion}

The performance of the algorithm is evaluated by the accuracy of eye location in the [8]. If the result meets the criteria, it is considered to be accurate. The accuracy rate formula is:

$$
\text { Accuracy rate }=\sum_{\substack{\mathrm{i}=1 \\ \mathrm{err}}<0.1}^{\mathrm{N}} 1 / N \times 100 \%
$$

Where $\boldsymbol{N}$ refers to the total number of face images used for the pupil center location, and $\boldsymbol{e r r}$ is the error of manual positioning of the pupil center and the locating pupil center by the algorithm.

In the experiment we select two groups face image database, Caltech face database (with different lighting and expression in the complex background) of 100 images and CVL face database (under uniform illumination, no flash and with simple background) of 100 images respectively. When the centers of the pupil of the two eyes are all located accurately, we think that 
the positioning is accurate. The accuracy rate result is shown in Table1.

Table1 shows that the average accuracy of pupil center is $92.45 \%$. Fig.13 shows some examples of accurate location images, which shows that the proposed algorithm can locate the center of the pupil in a complex condition, even if the face is tilted or has the interference of a spectacle. Fig.13 and table 1 show the effectiveness of the algorithm.

There are also some experiments failing in locating the pupil center, the reasons for the failure can be attributed to three: a) The light is too strong or too weak to detect face region; b) The pupil and the iris are occluded by the eyelid too much, which destroys the radial symmetry of the pupil and the iris; c) Eyes are kept out by hair too much, as a result the failure of eye region detection.

Table 1.Accuracy of pupil center location

$\begin{array}{lll}\text { Face database } & \text { Correct rate (\%) } & \text { Average correct rate (\%) } \\ \text { Caltech } & 92.59 & \\ \text { CVL } & 92.31 & 92.45\end{array}$

The results on Caltech
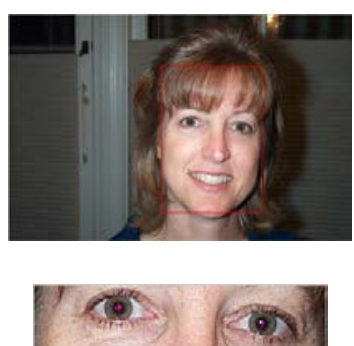

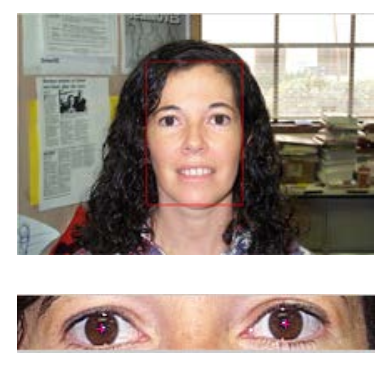

Fig.13.Puplil center location results

\section{Conclusions}

The results on CVL
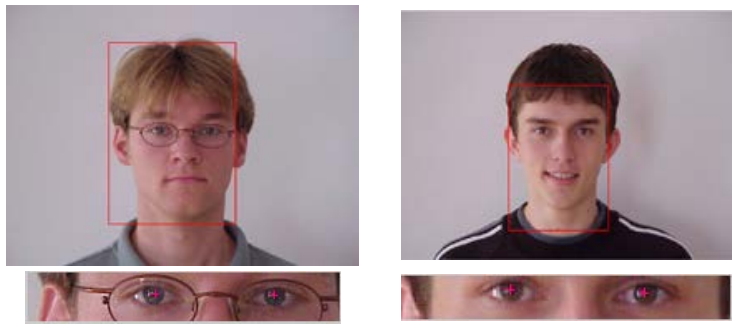

In this paper, a new algorithm for locating the pupil center in a color face image based on skin color segmentation and radial symmetry transform is proposed. The experimental results show that the presented method has high location accuracy and certain robustness. At the same time, the algorithm needs to be improved. In future work, plan to consider combining a light compensation method to solve the problem of uneven illumination which leads to face detection failure.

\section{Acknowledgements}

This work was financially supported by Projects of Education Department in Guangxi (201106LX020) and National College Students Innovation and Entrepreneurship Training Program.

\section{References}

[1] Jiannan Chi,Zhiliang Wang,Chuang Zhang, Line of Sight Tracking. Beijing:Publishing House of Machine Industry,2011:14.(In Chinese)

[2] Xiang Shulan,Cao Cheng,Aishy Amer,in: Journal of Southwest Jiaotong University(English Edition),2010,18(4):320:325

[3] Guofeng Zou, Kejun Wang,Tang Mo,in: IEEE,2013:1483-1486

[4] M.R. Fini, M.A.A. Kashani, in:IEEE, 2011:57-61

[5] R.L.Hsu, in: IEEE Trans.on Pattern Analysis and Machine Intelligence, 2002, 24(5):696-706

[6] R.C.Gonzalez,Digital Image Processing Using MARLAB. Beijing: Publishing House of Electronics Industry, 2013:261(In Chinese)

[7] G.Loy, in:IEEE Trans.on Pattern Analysis and Machine Intelligence,2003,25(8):959-972 
[8] O.Jesorsky, K.J. Kirchberg, R.W. Frischholz,in:International Conference on Audio and Video-Based Biometric Person Authentication, Springer, Halmstad, Sweden, 2011, 90-95 